ANNALES

POLONICI MATHEMATICI

$98.3(2010)$

\title{
Uniqueness of meromorphic functions sharing a meromorphic function of a smaller order with their derivatives
}

\author{
by XiaO-Min Li (Qingdao) and Hong-Xun Yi (Jinan)
}

\begin{abstract}
We prove some uniqueness theorems for meromorphic functions and their derivatives that share a meromorphic function whose order is less than those of the above meromorphic functions. The results in this paper improve those given by G. G. Gundersen \& L. Z. Yang, J. P. Wang, J. M. Chang \& Y. Z. Zhu, and others. Some examples are provided to show that our results are the best possible.
\end{abstract}

1. Introduction and main results. In this paper, by meromorphic functions we will always mean meromorphic functions in the complex plane. We adopt the standard notations of the Nevanlinna theory of meromorphic functions as explained in [6], [9] and [19]. It will be convenient to let $E$ denote any set of positive real numbers of finite linear measure, not necessarily the same at each occurrence. For a nonconstant meromorphic function $h$, we denote by $T(r, h)$ the Nevanlinna characteristic of $h$ and by $S(r, h)$ any quantity satisfying

$$
S(r, h)=o\{T(r, h)\} \quad(r \rightarrow \infty, r \notin E) .
$$

Let $f$ and $g$ be two nonconstant meromorphic functions, and let $a$ be a finite value. We say that $f$ and $g$ share the value a $C M$ provided that $f-a$ and $g-a$ have the same zeros with the same multiplicities. Similarly, we say that $f$ and $g$ share $a I M$ provided that $f-a$ and $g-a$ have the same zeros ignoring multiplicities. In addition, we say that $f$ and $g$ share $\infty C M$ if $1 / f$ and $1 / g$ share $0 \mathrm{CM}$, and we say that $f$ and $g$ share $\infty I M$ if $1 / f$ and $1 / g$ share 0 IM (see [20]).

In this paper, we also need the following definition.

Definition 1.1. For a nonconstant entire function $f$, the order $\sigma(f)$, the lower order $\mu(f)$, and the hyper order $\sigma_{2}(f)$ are defined as

2010 Mathematics Subject Classification: Primary 30D35, 30D30.

Key words and phrases: entire function, order of growth, shared value, uniqueness. 


$$
\begin{aligned}
\sigma(f) & =\limsup _{r \rightarrow \infty} \frac{\log T(r, f)}{\log r}=\limsup _{r \rightarrow \infty} \frac{\log \log M(r, f)}{\log r}, \\
\mu(f) & =\liminf _{r \rightarrow \infty} \frac{\log T(r, f)}{\log r}=\liminf _{r \rightarrow \infty} \frac{\log \log M(r, f)}{\log r}, \\
\sigma_{2}(f) & =\limsup _{r \rightarrow \infty} \frac{\log \log T(r, f)}{\log r}=\limsup _{r \rightarrow \infty} \frac{\log \log \log M(r, f)}{\log r}
\end{aligned}
$$

respectively; here and in what follows, $M(r, f)=\max _{|z|=r}|f(z)|$.

In 1977, L. A. Rubel and C. C. Yang [15] proved that if an entire function $f$ shares two distinct finite complex numbers CM with its derivative $f^{\prime}$, then $f=f^{\prime}$. What is the relation between $f$ and $f^{\prime}$ if an entire function $f$ shares one finite complex number $a \mathrm{CM}$ with its derivative $f^{\prime}$ ? In 1996, R. Brück [2] made the conjecture that if $f$ is a nonconstant entire function satisfying $\sigma_{2}(f)<\infty$, where $\sigma_{2}(f)$ is not a positive integer, and if $f$ and $f^{\prime}$ share one finite complex number $a \mathrm{CM}$, then $f-a=c\left(f^{\prime}-a\right)$ for some constant $c \neq 0$. He proved this for $a=0$. He also proved that the conjecture is true provided that $a \neq 0$ and $N\left(r, 1 / f^{\prime}\right)=S(r, f)$. In 2005, Al-Khaladi showed that the conjecture remains true for a nonconstant meromorphic function $f$ such that $N\left(r, 1 / f^{\prime}\right)=S(r, f)$ (see [1]). Recently many mathematicians have dealt with the above conjecture: A. Banerjee \& P. Bhattacharjee [3], J. M. Chang \& Y. Z. Zhu [4], J. Heittokangas et al. [7], I. Lahiri \& A. Sarkar [8], X. M. Li \& C. C. Gao [10], [11], X. M. Li \& H. X. Yi [12]-[14], J. Wang \& I. Laine [16], J. Wang \& X. M. Li [17], Q. C. Zhang [21], J. L. Zhang \& L. Z. Yang [22], [23], among others. But the conjecture is still an open question by now.

In 1998, G. G. Gundersen and L. Z. Yang proved the following result, which shows that the above conjecture is true for $a \neq 0$ provided that $f$ satisfies the additional assumption $\sigma(f)<\infty$.

TheOrem A (see [5, Theorem 1]). Let $f$ be a nonconstant entire function of finite order, and let $a(\neq 0)$ be a finite complex number. If $f$ and $f^{\prime}$ share a $C M$, then $f^{\prime}-a=c(f-a)$ for some nonzero constant $c$.

In 2004, J. P. Wang proved the following theorem, which improved Theorem A.

TheOREM B (see [18, Theorem 1]). Let $f$ be a nonconstant entire function of finite order, let $P$ be a polynomial with degree $p \geq 1$, and let $k$ be a positive integer. If $f-P$ and $f^{(k)}-P$ share $0 C M$, then $f^{(k)}-P=c(f-P)$ for some complex number $c \neq 0$.

Recently J. M. Chang and Y. Z. Zhu proved the following result, which improved Theorem B. 
Theorem C (see [4, Theorem 1]). Let $f$ be an entire function such that $\sigma(f)<\infty$, and let $a(\not \equiv 0)$ be an entire function such that $\sigma(a)<\sigma(f)$. If $f-a$ and $f^{\prime}-a$ share $0 C M$, then $f^{\prime}-a=c(f-a)$ for some nonzero constant $c$.

We will prove the following result, which improves Theorem C.

THEOREM 1.1. Let $f$ be a nonconstant entire function such that $\sigma(f)$ $<\infty$, and let $a(\not \equiv 0)$ be an entire function such that $\sigma(a)<\sigma(f)$. If $f-a$ and $f^{(k)}-a$ share $0 C M$, where $k(\geq 1)$ is a positive integer, then $f^{(k)}-a=c(f-a)$ for some nonzero constant $c$.

The following example shows that the condition " $\sigma(a)<\sigma(f)$ " in Theorem 1.1 is the best possible.

EXAmple 1.1 (see [4]). Let $f(z)=e^{2 z}-(z-1) e^{z}$ and $a(z)=e^{2 z}-z e^{z}$. Then it can be verified that $f-a$ and $f^{\prime}-a$ share $0 \mathrm{CM}$ and $\sigma(f)=\sigma(a)=1$. But $f^{\prime}-a=e^{z}(f-a)$.

In the same paper [4], J. M. Chang and Y. Z. Zhu prove the following result.

Theorem D (see [4, Theorem 2]). Let $f$ and a be meromorphic functions such that $f$ and a have finitely many poles, and such that $f$ and a have no common poles. If $f-a$ and $f^{\prime}-a$ share $0 C M$, and if $\sigma(a)<\sigma(f)<\infty$, then $f^{\prime}-a=c(f-a)$ for some nonzero constant $c$.

We will prove the following result, which improves Theorem D.

THEOREM 1.2. Let $f$ be a nonconstant meromorphic function, and let $a(\not \equiv 0)$ be a meromorphic function such that $f$ and a have finitely many poles, and such that $f$ and a have no common poles. If $\sigma(a)<\sigma(f)<\infty$, and if $f-a$ and $f^{(k)}-a$ share $0 C M$, where $k(\geq 1)$ is a positive integer, then $f^{(k)}-a=c(f-a)$ for some nonzero constant $c$.

Lemma 2.1 in Section 2 implies the following result, which complements Theorem 1.2.

THEOREM 1.3. Let $f$ be a nonconstant rational function, and let a be a nonzero complex number. If $f-a$ and $f^{(k)}-a$ share $0 C M$, where $k(\geq 1)$ is a positive integer, then $f$ can be expressed as

$$
f(z)=a+\frac{a\left(z-z_{1}\right)^{k}}{2 k !}+\frac{A_{1}}{z-z_{1}},
$$

where $z_{1}$ and $A_{1}(\neq 0)$ are two complex numbers.

The following example shows that the condition " $f$ and $a$ have finitely many poles" in Theorem 1.2 is the best possible. 
EXAmple 1.2 (see [5]). Let $f(z)=\left(2 e^{z}+z+1\right) /\left(e^{z}+1\right)$. Then $f$ is a nonconstant meromorphic function but not an entire function. Moreover, we can verify that $f$ and $f^{\prime}$ share $1 \mathrm{CM}$, that $\mu(f)=\sigma(f)=1$ and that $f$ has infinitely many poles. However, $\left(f^{\prime}-1\right) /(f-1)=-e^{z} /\left(e^{z}+1\right)$.

J. M. Chang and Y. Z. Zhu [4] prove the following result.

TheOREM E (see [4, Theorem 3]). Let $f$ be a nonconstant entire function of finite order. If $1 / f^{\prime}$ and $1 / f$ have the same fixed points with the same multiplicities, then $f=f^{\prime}$.

We will prove the following result, which improves Theorem E.

THEOREM 1.4. Let $f$ be a nonconstant entire function of finite order, and let $R$ be a nonzero rational function that has at least one pole. If $f-R$ and $f^{(k)}-R$ share $0 C M$, then $f=f^{(k)}$.

In 1995, H. X. Yi and C. C. Yang posed the following question.

QUestion 1.1 (see [20, p. 398]). Let $f$ be a nonconstant meromorphic function, and let $a$ be a finite nonzero complex constant. If $f, f^{(n)}$ and $f^{(m)}$ share the value $a \mathrm{CM}$, where $n$ and $m(n<m)$ are distinct positive integers not all even or odd, can we infer that $f=f^{(n)}$ ?

Regarding Question 1.1, G. G. Gundersen and L. Z. Yang proved the following result in 1998.

TheOREM $\mathrm{F}$ (see [5, Theorem 2]). Let $f$ be a nonconstant entire function of finite order, let $a(\neq 0)$ be a finite complex number, and let $n$ be a positive integer. If $a$ is shared by $f, f^{(n)}$ and $f^{(n+1)} I M$, and shared by $f^{(n)}$ and $f^{(n+1)}$ $C M$, then $f=f^{\prime}$.

We will prove the following result, which improves Theorem F.

THEOREM 1.5. Let $f$ be a nonconstant meromorphic function such that $f$ has finitely many poles and $\sigma(f)<\infty$, let $a(\neq 0)$ be a finite value in the complex plane, and let $n$ be a positive integer. If a is shared by $f, f^{(n)}$ and $f^{(n+1)} I M$, and shared by $f^{(n)}$ and $f^{(n+1)} C M$, then $f(z)=\gamma e^{z}$, where $\gamma$ is a certain nonzero constant.

\section{Some lemmas}

Lemma 2.1 (see [20, Corollary of Theorem 1.23]). Let $f$ be a nonconstant meromorphic function, and let $k(\geq 1)$ be a positive integer. Then $T(r, f) \leq \bar{N}(r, f)+N\left(r, \frac{1}{f}\right)+N\left(r, \frac{1}{f^{(k)}-1}\right)-N\left(r, \frac{1}{f^{(k+1)}}\right)+S(r, f)$.

Let $f=\sum_{n=0}^{\infty} a_{n} z^{n}$ be an entire function. We define by $\mu(r)=$ $\max \left\{\left|a_{n}\right| r^{n}: n=0,1,2, \ldots\right\}$ the maximum term of $f$, and define by $\nu(r, f)=$ $\max \left\{m: \mu(r)=\left|a_{m}\right| r^{m}\right\}$ the central index of $f$ (see [9, p. 50]). 
Lemma 2.2 (see [9, Theorem 3.1]). If $f$ is an entire function of order $\sigma(f)$, then

$$
\sigma(f)=\limsup _{r \rightarrow \infty} \frac{\log \nu(r, f)}{\log r} .
$$

Lemma 2.3 (see $[9$, p. 5]). Let $g:(0, \infty) \rightarrow \mathbb{R}$ and $h:(0, \infty) \rightarrow \mathbb{R}$ be increasing functions such that $g(r) \leq h(r)$ outside of an exceptional set $E_{1}$ of finite logarithmic measure. Then, for any $\alpha>1$, there exists $r_{0}>0$ such that $g(r) \leq h\left(r^{\alpha}\right)$ for all $r>r_{0}$.

Lemma 2.4 (see [9, Lemma 1.1.1]). Let $g:(0, \infty) \rightarrow \mathbb{R}$ and $h:(0, \infty) \rightarrow \mathbb{R}$ be increasing functions such that $g(r) \leq h(r)$ outside of an exceptional set $E$ of finite linear measure. Then, for any $\alpha>1$, there exists $r_{0}>0$ such that $g(r) \leq h(\alpha r)$ for all $r>r_{0}$.

Lemma 2.5 (see [20, Theorem 1.21]). Suppose that $f$ is meromorphic in the complex plane. Then $\sigma(f)=\sigma\left(f^{\prime}\right)$ and $\mu(f)=\mu\left(f^{\prime}\right)$.

\section{Proof of theorems}

Proof of Theorem 1.2. From the condition $\sigma(a)<\sigma(f)$ we know that $f$ is a transcendental meromorphic function. We discuss the following three cases.

CASE 1. Suppose that $f$ and $a$ are not entire functions. Then

$$
a=\frac{a_{1}}{P_{1}},
$$

where $P_{1}$ is a nonconstant polynomial such that $P_{1}$ and $1 / a$ share $0 \mathrm{CM}$. From the condition that $f-a$ and $f^{(k)}-a$ share $0 \mathrm{CM}$ we get

$$
\frac{f^{(k)}-a}{f-a}=\frac{c e^{\beta_{1}}}{\left(z-\omega_{1}\right)^{k} \cdots\left(z-\omega_{n}\right)^{k}},
$$

where $c$ is some nonzero complex number, $\omega_{1}, \ldots, \omega_{n}$ are $n$ distinct complex numbers that are all poles of $f$, and $\beta_{1}$ is an entire function. Let

$$
F=P_{0} f,
$$

where $P_{0}$ is a nonconstant polynomial such that $P_{0}$ and $1 / f$ share $0 \mathrm{CM}$. Then $F$ is a transcendental entire function. From (3.3) we get

$$
\begin{aligned}
\frac{f^{(k)}-a}{f-a}= & \frac{\frac{F^{(k)}}{F}+\frac{k R_{0}^{\prime}}{R_{0}} \cdot \frac{F^{(k-1)}}{F}+\cdots+\left(\begin{array}{c}
k \\
j
\end{array}\right) \frac{R_{0}^{(j)}}{R_{0}} \cdot \frac{F^{(k-j)}}{F}}{1-\frac{a P_{0}}{F}} \\
& +\frac{\left(\begin{array}{c}
k \\
j+1
\end{array}\right) \frac{R_{0}^{(j+1)}}{R_{0}} \cdot \frac{F^{(k-j-1)}}{F}+\cdots+\left(\begin{array}{c}
k \\
k-2
\end{array}\right) \frac{R_{0}^{(k-2)}}{R_{0}} \cdot \frac{F^{(2)}}{F}}{1-\frac{a P_{0}}{F}} \\
& +\frac{\frac{k R_{0}^{(k-1)}}{R_{0}} \cdot \frac{F^{\prime}}{F}+\frac{R_{0}^{(k)}}{R_{0}}-\frac{a P_{0}}{F}}{1-\frac{a P_{0}}{F}},
\end{aligned}
$$


where

$$
R_{0}=\frac{1}{P_{0}} .
$$

From (3.5) and the definition of $P_{0}$ we get

$$
\frac{R_{0}^{\prime}}{R_{0}}=\frac{m_{1}}{z-\omega_{1}}+\cdots+\frac{m_{n}}{z-\omega_{n}},
$$

where $m_{1}, \ldots, m_{n}$ are negative integers. By induction from (3.6) we get

$$
\frac{R_{0}^{(j)}(z)}{R_{0}(z)}=\frac{\left\{(-1)^{j-1}(j-1) ! \sum_{l=1}^{n} m_{l}\right\}(1+o(1))}{z^{j}}
$$

as $|z| \rightarrow \infty$, where $j$ is a positive integer satisfying $1 \leq j \leq k$. Noting that $F$ is a transcendental entire function, by Lemma 2.2 and Proposition 3.3 in [9] we know that

$$
\nu(r, F) \rightarrow \infty
$$

Let

$$
M(r, F)=\left|F\left(z_{r}\right)\right|,
$$

where $z_{r}=r e^{i \theta(r)}$ and $\theta(r) \in[0,2 \pi)$. From (3.9) and the Wiman-Valiron theory (see $\left[9\right.$, Theorem 3.2]), we know that there exists a subset $E_{j} \subset(1, \infty)$ with finite logarithmic measure, i.e., $\int_{E_{j}} d t / t<\infty$, such that for some point $z_{r}=r e^{i \theta(r)}(\theta(r) \in[0,2 \pi))$ satisfying $\left|z_{r}\right|=r \notin E_{j}$ and $M(r, F)=\left|F\left(z_{r}\right)\right|$, we have

$$
\frac{F^{(j)}\left(z_{r}\right)}{F\left(z_{r}\right)}=\left(\frac{\nu(r, F)}{z_{r}}\right)^{j}\{1+o(1)\}
$$

as $r \notin \bigcup_{j=1}^{k} E_{j}$ and $r \rightarrow \infty$. From (3.1) we see that $a_{1}$ is an entire function such that

$$
\sigma\left(a_{1} P_{0}\right)=\sigma\left(a_{1}\right)=\sigma(a) .
$$

From (3.3) we get $T(r, F)=T(r, f)+O(\log r)$. This together with (3.11) and the condition $\sigma(a)<\sigma(f)$ implies that

$$
\sigma\left(a_{1} P_{0}\right)<\sigma(F)
$$

By (3.12), Definition 1.1 and Lemma 2.3 we can deduce that there exists an infinite sequence of points $z_{r_{n}}=r_{n} e^{i \theta\left(r_{n}\right)}$ satisfying $M\left(r_{n}, F\right)=\left|F\left(z_{r_{n}}\right)\right|$, where $r_{n} \in I, I \subseteq \mathbb{R}^{+}$is a subset with logarithmic measure $m_{l}(I)=\infty$, such that

$$
\begin{aligned}
\lim _{r_{n} \rightarrow \infty} \frac{\log \log M\left(r_{n}, F\right)}{\log r_{n}} & =\sigma(F), \\
\lim _{r_{n} \rightarrow \infty} \frac{M\left(r_{n}, a_{1} P_{0}\right)}{M\left(r_{n}, F\right)} & =0 .
\end{aligned}
$$


Next we will show that $\beta_{2}$ is a constant. In fact, if $k=1$, then (3.4) can be rewritten as

$$
\frac{f^{\prime}-a}{f-a}=\frac{\frac{F^{\prime}}{F}+\frac{R_{0}^{\prime}}{R_{0}}-\frac{a P_{0}}{F}}{1-\frac{a P_{0}}{F}} .
$$

From (3.1), (3.7)-(3.10) and (3.13)-(3.15) we get

$$
\frac{f^{\prime}\left(z_{r_{n}}\right)-a\left(z_{r_{n}}\right)}{f\left(z_{r_{n}}\right)-a\left(z_{r_{n}}\right)}=\frac{\nu\left(r_{n}, F\right)}{z_{r_{n}}}\{1+o(1)\}
$$

as $r_{n} \in I \backslash E_{1}$ and $r_{n} \rightarrow \infty$. From (3.1) and (3.16) we get

$$
\begin{aligned}
\left|\beta_{1}\left(z_{r_{n}}\right)\right| & =\left|\log e^{\beta_{1}\left(z_{r_{n}}\right)}\right|=\left|\log \frac{f^{(k)}\left(z_{r_{n}}\right)-a\left(z_{r_{n}}\right)}{f\left(z_{r_{n}}\right)-a\left(z_{r_{n}}\right)}\right| \\
& \leq \log \left\{\nu\left(r_{n}, F\right)\right\}+\log r_{n}+O(1)
\end{aligned}
$$

as $r_{n} \in I \backslash E_{1}$ and $r_{n} \rightarrow \infty$. From the condition $\sigma(a)<\sigma(f)<\infty$ and (3.2) we know that there exists a sufficiently large positive number $r_{0}$ and a positive number $B_{1}$ such that

$$
T\left(r, e^{\beta_{1}}\right) \leq B_{1} r^{1+\sigma(f)} \quad\left(r \geq r_{0}\right),
$$

which implies that $\beta_{1}$ is a polynomial. From (3.17), Definition 1.1 and Lemma 2.2 we get

$$
\left|\beta_{1}\left(z_{r_{n}}\right)\right| \leq \log \left\{\nu\left(r_{n}, F\right)\right\}+\log r_{n}+O(1) \leq\{2+\sigma(f)\} \log r_{n}
$$

as $r_{n} \in I \backslash E_{1}$ and $r_{n} \rightarrow \infty$.

If $k \geq 2$, from (3.1), (3.4), (3.7)-(3.10), (3.13) and (3.14) we get

$$
\begin{aligned}
& \frac{f^{(k)}\left(z_{r_{n}}\right)-a\left(z_{r_{n}}\right)}{f\left(z_{r_{n}}\right)-a\left(z_{r_{n}}\right)} \\
&= \frac{\frac{F^{(k)}\left(z_{r_{n}}\right)}{F\left(z_{r_{n}}\right)}+\frac{k R_{0}^{\prime}\left(z_{r_{n}}\right)}{R_{0}\left(z_{r_{n}}\right)} \cdot \frac{F^{(k-1)\left(z_{r_{n}}\right)}}{F\left(z_{r_{n}}\right)}+\cdots+\left(\begin{array}{c}
k \\
j
\end{array}\right) \frac{R_{0}^{(j)}\left(z_{r_{n}}\right)}{R_{0}\left(z_{r_{n}}\right)} \cdot \frac{F^{(k-j)}\left(z_{r_{n}}\right)}{F\left(z_{r_{n}}\right)}}{1-\frac{a\left(z_{r_{n}}\right) P_{0}\left(z_{r_{n}}\right)}{F\left(z_{r_{n}}\right)}} \\
&+\frac{\left(\begin{array}{c}
k \\
j+1
\end{array}\right) \frac{R_{0}^{(j+1)}\left(z_{r_{n}}\right)}{R_{0}\left(z_{r_{n}}\right)} \cdot \frac{F^{(k-j-1)}\left(z_{r_{n}}\right)}{F\left(z_{r_{n}}\right)}+\cdots+\left(\begin{array}{c}
k \\
k-2
\end{array}\right) \frac{R_{0}^{(k-2)}\left(z_{r_{n}}\right)}{R_{0}\left(z_{r_{n}}\right)} \cdot \frac{F^{(2)}\left(z_{r_{n}}\right)}{F\left(z_{r_{n}}\right)}}{1-\frac{a\left(z_{r_{n}}\right) P_{0}\left(z_{r_{n}}\right)}{F\left(z_{r_{n}}\right)}} \\
&+\frac{\frac{k R_{0}^{(k-1)}\left(z_{r_{n}}\right)}{R_{0}\left(z_{r_{n}}\right)} \cdot \frac{F^{\prime}\left(z_{r_{n}}\right)}{F\left(z_{r_{n}}\right)}+\frac{R_{0}^{(k)}\left(z_{r_{n}}\right)}{R_{0}\left(z_{r_{n}}\right)}-\frac{a\left(z_{r_{n}}\right) P_{0}\left(z_{r_{n}}\right)}{F\left(z_{r_{n}}\right)}}{1-\frac{a\left(z_{r_{n}}\right) P_{0}\left(z_{r_{n}}\right)}{F\left(z_{r_{n}}\right)}} \\
&+\frac{N_{n} \sum_{j=1}^{k-1}\left(\begin{array}{c}
k \\
j
\end{array}\right) \sum_{j=1}^{k-1}(-1)^{j-1}(j-1) !\left\{\nu\left(r_{n}, F\right)\right\}^{k-j}\{1+o(1)\}}{z_{r_{n}}^{k}}=\left(\frac{\nu\left(r_{n}, F\right)}{z_{r_{n}}}\right)^{k}\{1+o(1)\} \\
& z_{r_{n}}^{k}
\end{aligned}
$$


as $r_{n} \in I \backslash \bigcup_{j=1}^{k} E_{j}$ and $r_{n} \rightarrow \infty$, where $N_{n}=\sum_{l=1}^{n} m_{l}$. Next in the same manner as in the proof of (3.18) we get

$$
\left|\beta_{1}\left(z_{r_{n}}\right)\right| \leq k\left\{\log \left\{\nu\left(r_{n}, F\right)\right\}+\log r_{n}\right\}+O(1) \leq k\{2+\sigma(f)\} \log r_{n}
$$

as $r_{n} \in I \backslash \bigcup_{j=1}^{k} E_{j}$ and $r_{n} \rightarrow \infty$. From (3.18) and (3.20) we know that $\beta_{1}$ is a constant. Thus (3.2) can be rewritten as

$$
\frac{f^{(k)}-a}{f-a}=\frac{c}{\left(z-\omega_{1}\right)^{k} \cdots\left(z-\omega_{n}\right)^{k}},
$$

where $c$ is some nonzero constant. From (3.7) we get

$$
\left|\frac{R_{0}^{(j)}(z)}{R_{0}(z)}\right| \leq \frac{d_{j}}{|z|^{j}}
$$

as $|z| \rightarrow \infty$, where $j$ is a positive integer satisfying $1 \leq j \leq k, d_{j}$ is some positive constant. We discuss the following two subcases.

Subcase 1.1. Suppose $k=1$. Then (3.4) can be rewritten as (3.15). From (3.15) and (3.21) we get

$$
\frac{\frac{F^{\prime}}{F}+\frac{R_{0}^{\prime}}{R_{0}}-\frac{a P_{0}}{F}}{1-\frac{a P_{0}}{F}}=\frac{c}{\left(z-\omega_{1}\right) \cdots\left(z-\omega_{n}\right)} .
$$

From (3.10), (3.13) (3.14), (3.23) we get

$$
\begin{aligned}
\left|\frac{\nu\left(r_{n}, F\right)}{2 z_{r_{n}}}\right| \leq & \frac{|c|}{\left|z_{r_{n}}-\omega_{1}\right| \cdots\left|z_{r_{n}}-\omega_{n}\right|} \\
& +\frac{|c|}{\left|z_{r_{n}}-\omega_{1}\right| \cdots\left|z_{r_{n}}-\omega_{n}\right|} \cdot\left|\frac{a\left(z_{r_{n}}\right) P_{0}\left(z_{r_{n}}\right)}{F\left(z_{r_{n}}\right)}\right| \\
& +\left|\frac{a\left(z_{r_{n}}\right) P_{0}\left(z_{r_{n}}\right)}{F\left(z_{r_{n}}\right)}\right|+\left|\frac{R_{0}^{\prime}\left(z_{r_{n}}\right)}{R_{0}\left(z_{r_{n}}\right)}\right|
\end{aligned}
$$

as $r_{n} \in I \backslash E_{1}$ and $r_{n} \rightarrow \infty$. This together with (3.6) implies

$$
\begin{aligned}
\nu\left(r_{n}, F\right) \leq & \frac{2|c|\left|z_{r_{n}}\right|}{\left|z_{r_{n}}-\omega_{1}\right| \cdots\left|z_{r_{n}}-\omega_{n}\right|} \\
& +\frac{2|c|}{\left|z_{r_{n}}-\omega_{1}\right| \cdots\left|z_{r_{n}}-\omega_{n}\right|} \cdot\left|\frac{z_{r_{n}} a\left(z_{r_{n}}\right) P_{0}\left(z_{r_{n}}\right)}{F\left(z_{r_{n}}\right)}\right| \\
& +2\left|\frac{z_{r_{n}} a\left(z_{r_{n}}\right) P_{0}\left(z_{r_{n}}\right)}{F\left(z_{r_{n}}\right)}\right|+\left|\frac{2 z_{r_{n}} R_{0}^{\prime}\left(z_{r_{n}}\right)}{R_{0}\left(z_{r_{n}}\right)}\right| \\
\leq & 3\left|\frac{z_{r_{n}} a\left(z_{r_{n}}\right) P_{0}\left(z_{r_{n}}\right)}{F\left(z_{r_{n}}\right)}\right|+O(1)
\end{aligned}
$$

as $r_{n} \in I \backslash E_{1}$ and $r_{n} \rightarrow \infty$. From (3.1) we get

$$
\sigma\left(z a_{1} P_{0}\right)=\sigma\left(a_{1}\right)=\sigma(a) .
$$


From (3.25) and the condition $\sigma(a)<\sigma(F)$ we get

$$
\sigma\left(z a_{1} P_{0}\right)<\sigma(F) .
$$

From (3.1), (3.8) and (3.24) we get

$$
\begin{aligned}
\nu\left(r_{n}, F\right) & \leq 3\left|\frac{z_{r_{n}} a_{1}\left(z_{r_{n}}\right) P_{0}\left(z_{r_{n}}\right)}{P_{1}\left(z_{r_{n}}\right) F\left(z_{r_{n}}\right)}\right|+O(1) \\
& \leq 4\left|\frac{z_{r_{n}} a_{1}\left(z_{r_{n}}\right) P_{0}\left(z_{r_{n}}\right)}{F\left(z_{r_{n}}\right)}\right|
\end{aligned}
$$

as $r_{n} \in I \backslash E_{1}$ and $r_{n} \rightarrow \infty$. Thus

$$
\nu\left(r_{n}, F\right)\left|F\left(z_{r_{n}}\right)\right| \leq 4\left|z_{r_{n}} a_{1}\left(z_{r_{n}}\right) P_{0}\left(z_{r_{n}}\right)\right| \leq 4 M\left(r_{n}, z a_{1} P_{0}\right)
$$

as $r_{n} \in I \backslash E_{1}$ and $r_{n} \rightarrow \infty$. From (3.13), (3.28) and Definition 1.1 we know that

$$
\nu\left(r_{n}, F\right) e^{r_{n}^{\sigma(F)-\varepsilon}} \leq 4 M\left(r_{n}^{\alpha}, z a_{1} P_{0}\right) \leq e^{r_{n}^{\delta_{1}+\varepsilon}}
$$

as $r_{n} \in I \backslash E_{1}$ and $r_{n} \rightarrow \infty$, where

$$
\delta_{1}=\sigma\left(z a_{1} P_{0}\right)
$$

and $\varepsilon$ is an arbitrary positive number. From (3.8), (3.26), (3.29) and (3.30) we get a contradiction.

SubCase 1.2. Suppose that

$$
k \geq 2 .
$$

From (3.4) and (3.21) we get

$$
\begin{aligned}
\frac{\frac{F^{(k)}}{F}+\frac{k R_{0}^{\prime}}{R_{0}} \cdot \frac{F^{(k-1)}}{F}+\cdots+\left(\begin{array}{c}
k \\
j
\end{array}\right) \frac{R_{0}^{(j)}}{R_{0}} \cdot \frac{F^{(k-j)}}{F}}{1-\frac{a P_{0}}{F}} & \\
+ & \frac{\left(\begin{array}{c}
k \\
j+1
\end{array}\right) \frac{R_{0}^{(j+1)}}{R_{0}} \cdot \frac{F^{(k-j-1)}}{F}+\cdots+\left(\begin{array}{c}
k \\
k-2
\end{array}\right) \frac{R_{0}^{(k-2)}}{R_{0}} \cdot \frac{F^{(2)}}{F}}{1-\frac{a P_{0}}{F}} \\
& +\frac{\frac{k R_{0}^{(k-1)}}{R_{0}} \cdot \frac{F^{\prime}}{F}+\frac{R_{0}^{(k)}}{R_{0}}-\frac{a P_{0}}{F}}{1-\frac{a P_{0}}{F}} \\
= & \frac{c}{\left(z-\omega_{1}\right)^{k} \cdots\left(z-\omega_{n}\right)^{k}} .
\end{aligned}
$$


From (3.10), (3.13), (3.14), (3.22) and (3.32) we get

$$
\begin{aligned}
\{\nu & \left.\left(r_{n}, F\right)\right\}^{k} \\
\leq & 4\left\{k\left|d_{1}\right|\left\{\nu\left(r_{n}, F\right)\right\}^{k-1}+\cdots+\left(\begin{array}{c}
k \\
j
\end{array}\right)\left|d_{j}\right|\left\{\nu\left(r_{n}, F\right)\right\}^{k-j}+\cdots\right. \\
& \left.+k\left|d_{k-1}\right| \nu\left(r_{n}, F\right)+\left|d_{k}\right|+\frac{\left|a_{1}\left(z_{r_{n}}\right) z_{r_{n}}^{k} P_{0}\left(z_{r_{n}}\right)\right|}{\left|P_{1}\left(z_{r_{n}}\right) F\left(z_{r_{n}}\right)\right|}\right\} \\
& +\frac{|c|\left|z_{r_{n}}^{k}\right|}{\left|z_{r_{n}}-\omega_{1}\right| k \cdots\left|z_{r_{n}}-\omega_{n}\right|^{k}} \cdot\left\{1+\frac{\left|a_{1}\left(z_{r_{n}}\right) P_{0}\left(z_{r_{n}}\right)\right|}{\left|P_{1}\left(z_{r_{n}}\right) F\left(z_{r_{n}}\right)\right|}\right\} \\
& \leq 4\left\{k\left|d_{1}\right|\left\{\nu\left(r_{n}, F\right)\right\}^{k-1}+\cdots+\left(\begin{array}{c}
k \\
j
\end{array}\right)\left|d_{j}\right|\left\{\nu\left(r_{n}, F\right)\right\}^{k-j}+\cdots\right. \\
& \left.+k\left|d_{k-1}\right| \nu(r, F)+\left|d_{k}\right|+\frac{\left|z_{r_{n}}^{k} a_{1}\left(z_{r_{n}}\right) P_{0}\left(z_{r_{n}}\right)\right|}{\left|F\left(z_{r_{n}}\right)\right|}\right\} \\
& +2|c|\left\{1+\frac{\left|a_{1}\left(z_{r_{n}}\right) P_{0}\left(z_{r_{n}}\right)\right|}{\left|F\left(z_{r_{n}}\right)\right|}\right\}
\end{aligned}
$$

as $r_{n} \in I \backslash \bigcup_{j=1}^{k} E_{j}$ and $r_{n} \rightarrow \infty$. From (3.8) and (3.33) we get

$$
\left\{\nu\left(r_{n}, F\right)\right\}^{k} \leq \frac{16\left|z_{r_{n}}^{k} a_{1}\left(z_{r_{n}}\right) P_{0}\left(z_{r_{n}}\right)\right|}{\left|F\left(z_{r_{n}}\right)\right|}+O(1) \leq \frac{17\left|z_{r_{n}}^{k} a_{1}\left(z_{r_{n}}\right) P_{0}\left(z_{r_{n}}\right)\right|}{\left|F\left(z_{r_{n}}\right)\right|}
$$

as $r_{n} \in I \backslash \bigcup_{j=1}^{k} E_{j}$ and $r_{n} \rightarrow \infty$. From (3.34) we get

$$
\left\{\nu\left(r_{n}, F\right)\right\}^{k}\left|F\left(z_{r_{n}}\right)\right| \leq 17\left|z_{r_{n}}^{k} a_{1}\left(z_{r_{n}}\right) P_{0}\left(z_{r_{n}}\right)\right| \leq 17 M\left(r_{n}, z^{k} a_{1} P_{0}\right)
$$

as $r_{n} \in I \backslash \bigcup_{j=1}^{k} E_{j}$ and $r_{n} \rightarrow \infty$. From (3.1) and (3.3) we get $\sigma\left(a_{1}\right)=\sigma(a)$ and $\sigma(F)=\sigma(f)$ respectively. This together with $\sigma\left(z^{k} a_{1} P_{0}\right)=\sigma\left(a_{1}\right)$ and the condition $\sigma(a)<\sigma(f)$ gives

$$
\delta_{2}<\sigma(F),
$$

where

$$
\delta_{2}=\sigma\left(z^{k} a_{1} P_{0}\right) .
$$

From (3.13), (3.35), (3.37) and Definition 1.1 we get

$$
\left\{\nu\left(r_{n}, F\right)\right\}^{k} e^{r_{n}^{\sigma(F)-\varepsilon}} \leq 17 e^{r_{n}^{\delta_{2}+\varepsilon}}
$$

as $r_{n} \in I \backslash \bigcup_{j=1}^{k} E_{j}$ and $r_{n} \rightarrow \infty$. From (3.8), (3.36) and (3.38) we get a contradiction.

Case 2. Suppose that $f$ is not an entire function and $a$ is an entire function. Proceeding as in Case 1 we get contradictions.

Case 3. Suppose that $f$ is an entire function. Then from the condition that $f-a$ and $f^{(k)}-a$ share $0 \mathrm{CM}$ we get 


$$
\frac{f^{(k)}-a}{f-a}=e^{\beta_{2}}
$$

where $\beta_{2}$ is an entire function. Proceeding as in Case 1 we deduce from (3.39) that $\beta_{2}$ is a constant, which yields the conclusion of Theorem 1.2.

Theorem 1.2 is thus completely proved.

Proof of Theorem 1.1. By the assumptions of Theorem 1.1 we have (3.39). Next in the same manner as in Case 1 of the proof of Theorem 1.2 we get the conclusion of Theorem 1.1.

Proof of Theorem 1.4. By the assumptions of Theorem 1.4 we have

$$
\frac{f^{(k)}-R}{f-R}=e^{\beta_{3}}
$$

where $\beta_{3}$ is an entire function. If $f$ is a polynomial, then it follows from (3.40) that $\beta_{3}$ is a constant. If $e^{\beta_{3}}=1$, from (3.40) we get the conclusion of Theorem 1.4. Next we suppose that $e^{\beta_{3}} \neq 1$. By rewriting (3.40) we get

$$
f^{(k)}-f e^{\beta_{3}}=R\left(1-e^{\beta_{3}}\right) .
$$

From (3.41) and the supposition that $R$ has at least one pole we get a contradiction. Next we suppose that $f$ is a transcendental entire function. Proceeding as in Case 1 of the proof of Theorem 1.1 we infer from (3.41) and the condition $\sigma(f)<\infty$ that $\beta_{3}$ is a polynomial. If $\beta_{3}$ is not constant, from (3.41) we get

$$
T\left(r, e^{\beta_{3}}\right) \leq 2 T(r, f)+O\{\log T(r, f)+\log r\} \quad(r \in I \backslash E) .
$$

From (3.42) and Lemma 2.4 we know that there exists a sufficiently large positive number $r_{0}$ such that

$$
T\left(r, e^{\beta_{3}}\right) \leq 2 T(2 r, f)+O\{\log T(2 r, f)+\log r+\log 2\} \quad\left(r \geq r_{0}\right) .
$$

From (3.43) and Definition 1.1 we get

$$
1 \leq \operatorname{deg}\left(\beta_{3}\right)=\sigma\left(e^{\beta_{3}}\right) \leq \sigma(f) .
$$

From (3.44) and $\sigma(R)=0$ we get

$$
\sigma(R)<\sigma(f) .
$$

From (3.45) and Theorem 1.2 we find that $e^{\beta_{3}}$ is a constant, which contradicts the above supposition. Next we suppose that $\beta_{3}$ is a constant. If $e^{\beta_{3}} \neq 1$, by rewriting (3.40) as (3.41) and using the condition that $R$ has at least one pole we get a contradiction. Thus $e^{\beta_{3}}=1$. This together with (3.40) yields the conclusion of Theorem 1.4.

Proof of Theorem 1.5. We discuss the following two cases.

CASE 1. Suppose that $f$, and so $f^{(n)}$, is a nonconstant rational function. Then from Theorem 1.3 and the condition that $f^{(n)}$ and $f^{(n+1)}$ share $a$ CM 
we get

$$
f^{(n)}(z)=a+\frac{a\left(z-z_{1}\right)}{2}+\frac{A_{1}}{z-z_{1}},
$$

where $z_{1}$ and $A_{1}(\neq 0)$ are two complex numbers. From (3.46) we get a contradiction.

CAse 2. Suppose that $f$ is a transcendental meromorphic function. Then from Lemma 2.5 we get

$$
\sigma(f)=\sigma\left(f^{\prime}\right)
$$

Similarly

$$
\sigma\left(f^{(j)}\right)=\sigma\left(f^{j+1}\right) \quad(1 \leq j \leq n-1) .
$$

From (3.47) and (3.48) we get

$$
\sigma(f)=\sigma\left(f^{(n)}\right) .
$$

From (3.49) and the condition $\sigma(f)<\infty$ we get

$$
\sigma\left(f^{(n)}\right)<\infty \text {. }
$$

From (3.50), Theorem 1.2 and the condition that $f^{(n)}$ and $f^{(n+1)}$ share $a$ $\mathrm{CM}$ we get

$$
f^{(n+1)}-a=c\left(f^{(n)}-a\right),
$$

where $c$ is some nonzero constant. From (3.51) we know that $f$ is a nonconstant entire function. This together with Theorem F yields the conclusion of Theorem 1.5.

Acknowledgements. The authors wish to express their thanks to the referee for his/her valuable suggestions and comments.

This project was supported by the NSFC (No. 10771121), the NSFC \& RFBR (Joint Project) (No. 10911120056), the NSF of Shandong Province, China (No. Z2008A01), and the NSF of Shandong Province, China (No. ZR2009AM008).

\section{References}

[1] A. Al-Khaladi, On meromorphic functions that share one value with their derivative, Analysis 25 (2005), 131-140.

[2] R. Brück, On entire functions which share one value CM with their first derivative, Results Math. 30 (1996), 21-24.

[3] A. Banerjee and P. Bhattacharjee, Uniqueness of meromorphic functions sharing one value with their derivatives, Math. Comm. 13 (2008), 277-288.

[4] J. M. Chang and Y. Z. Zhu, Entire functions that share a small function with their derivatives, J. Math. Anal. Appl. 351 (2009), 491-496.

[5] G. G. Gundersen and L. Z. Yang, Entire functions that share one value with one or two of their derivatives, ibid. 223 (1998), 88-95. 
[6] W. K. Hayman, Meromorphic Functions, Clarendon Press, Oxford, 1964.

[7] J. Heittokangas, R. Korhonen, I. Laine, J. Rieppo and J. Zhang, Value sharing results for shifts of meromorphic functions and sufficient conditions for periodicity, J. Math. Anal. Appl. 355 (2009), 352-363.

[8] I. Lahiri and A. Sarkar, Uniqueness of a meromorphic function and its derivative, J. Inequal. Pure Appl. Math. 5 (2004), no 1, art. 20.

[9] I. Laine, Nevanlinna Theory and Complex Differential Equations, de Gruyter, Berlin, 1993.

[10] X. M. Li and C. C. Gao, On a kth-order differential equation, Ann. Polon. Math. 89 (2006), 53-63.

[11] - - -, Entire functions sharing one polynomial with their derivatives, Proc. Indian Acad. Sci. (Math Sci.) 118 (2008), 13-26.

[12] X. M. Li and H. X. Yi, An entire function and its derivatives sharing a polynomial, J. Math. Anal. Appl. 330 (2007), 66-79.

[13] - - -, On uniqueness of an entire function and its derivatives, Arch. Math. (Basel) 89 (2007), 216-225.

[14] -, - Some results on the regular solutions of a linear differential equation, Comput. Math. Appl. 56 (2008), 2210-2221.

[15] L. Rubel and C. C. Yang, Values shared by an entire function and its derivative, in: Complex Analysis (Kentucky 1976), Lecture Notes in Math. 599, Springer, Berlin, 1977, 101-103.

[16] J. Wang and I. Laine, Uniqueness of entire functions and their derivatives, Comput. Methods Function Theory 8 (2008), 327-338.

[17] J. Wang and X. M. Li, The uniqueness of an entire function sharing a small entire function with its derivatives, J. Math. Anal. Appl. 354 (2009), 478-489.

[18] J. P. Wang, Entire functions that share a polynomial with one of their derivatives, Kodai Math. J. 27 (2004), 144-151.

[19] L. Yang, Value Distribution Theory, Springer, Berlin, 1993.

[20] C. C. Yang and H. X. Yi, Uniqueness Theory of Meromorphic Functions, Kluwer, Dordrecht, 2003.

[21] Q. C. Zhang, Meromorphic function that shares one small function with its derivative, J. Inequal. Pure Appl. Math. 6 (2005), no. 4, art. 116.

[22] J. L. Zhang and L. Z. Yang, Some results related to a conjecture of R. Brück, ibid. 8 (2007), no. 1, art. 18.

[23] - - - Some results related to a conjecture of $R$. Brück concerning meromorphic functions sharing one small function with their derivatives, Ann. Acad. Sci. Fenn. Math. 32 (2007), 141-149.

Xiao-Min Li

Department of Mathematics

Ocean University of China

Qingdao, Shandong 266071

People's Republic of China

E-mail: xmli01267@gmail.com
Hong-Xun Yi

Department of Mathematics

Shandong University Jinan, Shandong 250100

People's Republic of China E-mail: hxyi@sdu.edu.cn

Received 19.5.2009

and in final form 20.10.2009 
